\title{
Enriching the Functionally Graded Materials (FGM) Ontology for Digital Manufacturing
}

\author{
Munira Mohd Ali ${ }^{a}$, Ruoyu Yang ${ }^{a}$, Binbin Zhang ${ }^{a}$, Francesco Furini ${ }^{b}$, \\ Rahul Rai ${ }^{a, *}$, J. Neil Otte ${ }^{c}$, Barry Smith ${ }^{d}$ \\ ${ }^{a}$ Department of Mechanical and Aerospace Engineering, State University of New York at \\ Buffalo, Buffalo, NY, 14260 \\ ${ }^{b}$ Politecnico di Milano, Piazza Leonardo da Vinco, 32 Milan, Italy 20133 \\ ${ }^{c}$ Applied Physics Laboratory, Johns Hopkins University, Laurel, MD, 21045 \\ ${ }^{d}$ National Center for Ontology Research, State University of New York at Buffalo, Buffalo, \\ NY, 14260 \\ *Corresponding author. Email: rahulrai@buffalo.edu
}

\begin{abstract}
Functionally graded materials (FGMs) have been used in many different kinds of applications in recent years and have attracted significant research attention. However, we do not yet have a commonly accepted way of representing the various aspects of FGMs. Lack of standardized vocabulary creates obstacles to the extraction of useful information relating to pertinent aspects of different applications. A standard resource is needed for describing various elements of FGMs, including existing applications, manufacturing techniques, and material characteristics. This motivated the creation of the FGM Ontology (FGMO) in 2016. Here, we present a revised and expanded version of the FGM Ontology, which includes enrichments along four dimensions: (1) documenting recent FGMs applications; (2) reorganizing the framework to incorporate an updated representation of types of manufacturing processes; (3) enriching the axioms of the ontology; and (4) importing midlevel ontologies from the Common Core Ontologies (CCO) and Product Life Cycle (PLC) Ontologies. The work is being carried out within the framework of the Industry Ontology Foundry (IOF), and the ontology is conformant to Basic Formal Ontology (BFO).
\end{abstract}

Keywords: FGM manufacturing ontology, Material ontology, Manufacturing process ontology, Ontology Engineering, Digital manufacturing.

\section{Introduction}

Functionally graded materials (FGMs) are composite materials in which mechanical, chemical, thermal, and physical properties vary continuously. FGMs were first introduced in Japan in 1984 as part of a project involving materials used in thermal barriers [1]. FGMs are used to manufacture components formed by particles of at least 
two different kinds of materials. The mixing gradient of different materials in FGM is specified by a mathematical function that depends on spatial coordinates along given axes. The sharp interfaces between fibers of conventional composite materials are replaced by smooth transitions ensured by the distribution of particles of the different constituent materials. An FGM is thus a kind of heterogeneous material that possesses the characteristics of multiple components simultaneously. As a result, FGMs have found wide applications in many areas, from medical implants to aerospace engineering, where the spatial gradation in structure and composition lends itself to the tailoring of specific combinations of material properties.

Since they were introduced in 1984, extensive research has been devoted to the study of FGMs, and interest in this area continues to increase. However, this very proliferation of research efforts poses problems. One of these is that the relationships among both the different studies and the various underlying domains of application are often unclear because relevant research data are stored in multiple heterogeneous ways. The data are dispersed as regards not only the different domains of application, but also different manufacturing techniques, material characteristics, and so forth.

An ontology is used to provide a common information structure that can support interoperability across heterogeneous data. The FGM Ontology (FGMO) [2] was introduced to counteract some of the effects of the lack of alignment and the resultant poor discoverability of FGM research data by providing a controlled, structured vocabulary, that could be used to tag such data consistently along the lines pioneered by ontology initiatives in other areas. The FGMO is designed to serve not only to provide the basis for more systematic information management but also to support the use of computational tools to reason with extracted FGM information. Imagine, for example, that we have a vast repository of FGM-relevant data sources and need to place queries concerning the FGMs used in particular applications. The use of the ontology to tag the data will help to ensure that the relevant materials, attributes, and manufacturing processes will be retrieved within a consistent and easily accessible framework [3]. The present release revises and expands this first version of the FGMO by taking into account the most recent documentation concerning new FGM applications and incorporating a revised taxonomy of manufacturing processes.

\section{Related Work}

\subsection{Ontology in engineering}

Ontology has its origins as a subfield of philosophy focusing (roughly) on the study of the higher-order categories of being that make up reality - in other words, categories such as object, process, quality, function, and the relations among them. Today, ontology is also a field in the domain of information and computer science, where it studies the entities and relationships in particular domains to define general and shared information structures that promote interoperability among heterogeneous sources of data. 
The field of study concerned with applications of ontology within particular domains such as medicine or engineering is called applied ontology, and the activity involved in such use is termed ontology engineering (OE). Ontology engineers work to create and manage large-scale representations of entities such as actions, temporal intervals, physical objects, information artifacts, and so forth. Domain ontologies start from representations of categories of entities at very high levels of generality, which are then used by engineers as the starting point for creating representations of entities in specific domains through the specialization of general terms. For example, the term 'object' is specialized to form the term 'valve', which is then specialized further to 'ball valve', to 'single-body ball valve', and so on. In this way, a coordinated set of vocabularies is created that allows independent researchers to annotate data in a way that facilitates communication across multiple disciplinary groups [4]. In recent years, scientists working in $\mathrm{OE}$ have focused on applications in industrial engineering and related areas with the goal of creating re-usable and intuitive representations that allow researchers to manage, store, retrieve, and reuse their data effectively.

\subsection{Ontology in manufacturing and material engineering}

Manufacturing engineering is a critical domain in which OE has already made significant progress. For instance, in the work of Lemaignan et al. [5], the authors propose a new top-level ontology entitled MASON, for 'Manufacturing Semantics Ontology', designed to support semantic integration in the domain of manufacturing. MASON and has been applied in areas such as automatic cost estimation and multi-agent systems for manufacturing. MASON is then reused as one of the top-level ontology for the CREMA Data Model, Core Module Ontology (CDM-Core) [6], a manufacturing ontology that includes both the general manufacturing domain applicability and the specific project use cases. Chang et al. [7] develop the Design for Manufacturing (DFM) Ontology to represent information related to the design and manufacturing domains. With the DFM Ontology, all retrieved information from different feasible approaches in manufacturing a product will be able to assist the designer in selecting the best design approach based on the technical or economics requirements. Ameri et al. [8] introduce the Manufacturing Service Description Language (MSDL) to support the semantic framework for representing conventional manufacturing processes that analyze the manufacturing capabilities across the suppliers, workstations, machines, devices, and processes. Usman et al. [9] develop the Manufacturing Core Concepts Ontology (MCCO) that emphasizes interoperability across the production and design domains of the product life cycle. Similarly, Garetti et al. [10] propose a set of comprehensive definitions for terms representing different types of entities in the manufacturing domain, forming what is called the P-PSO, or Politecnico di Milano Production Systems Ontology. The purpose of the P-PSO is to facilitate the exchange of information about design and control activities in a way that provides the beginnings of a framework to formalize manufacturing information. 
Meanwhile, existing ontologies in the field of materials engineering include the ontology for metallic materials [11] and ontology for industrial materials [12]. Zhang et al. [11] constructed the ontology for metallic materials by applying a string matching algorithm to a large open knowledge base of metallic materials to enable the generation of ontology content from heterogeneous data. Cheung et al. [12] created the ontology for the industrial material, 'MatOnto', with the goal of representing materials of all kinds, especially in their raw state.

Unfortunately, these mentioned ontologies, like many similar ontologies developed in recent decades, were constructed in ad hoc ways. Little effort was spent to reuse the existing ontologies or to create a global framework of reference ontologies that could be applied and tested over and over again in different contexts and by different organizations to advance data interoperability in their respective fields. For example, the definitions proposed in [11] are not precise enough to create an ontology that could be recommended for reuse. MatOnto [12] remained dormant for some years due to its little practical utility in its initial implementation. Therefore, there is an initiative coordinated by the US National Institute of Standards and Technology (NIST) that aims to promote the reuse of standard reference ontologies. This initiative is the Industrial Ontologies Foundry (IOF) [13] whose mission is to provide an open-source platform for developing, validating, aligning, sharing, and curating industrial ontologies.

\subsection{Industrial Ontologies Foundry (IOF)}

One of the technical goals of IOF is to create a suite of high-quality, interoperable ontologies that covers the entire domain of digital manufacturing [13]. The ontologies should remain generic for it to be easily reused and extended to derive other domaindependent or application ontologies.

To promote interoperability of ontologies developed in the IOF, the ontologies are connected together in a multi-tiered hub and spokes structure incorporating [14]:

1. A single, small, domain-neutral top-level ontology;

2. Mid-level ontologies covering broad domains having root nodes that are either direct children of classes from the top-level ontology or of a class drawn from another mid-level ontology within the network;

3. Lower-level ontologies representing specialized domains having root nodes that are either direct children of classes from one of the mid-level ontologies or of a class drawn from another domain level ontology within the network.

Basic Formal Ontology (BFO $)^{1}$ has been adopted as the top-level ontology for the ontologies developed in the IOF. BFO and Descriptive Ontology for Linguistic and

\footnotetext{
${ }^{1}$ See https://github.com/BFO-ontology/BFO/blob/master/bfo.owl
} 
Cognitive Engineering (DOLCE) are two commonly used top-level ontologies that have been used as foundation ontologies for domain ontology development [15] [16] [17]. Both of them grew out of a similar philosophical orientation but adopted different approaches to ontological details [15]. However, BFO has shown a greater capacity to serve as the basis of sustainable suites of modular ontologies structured in the aforementioned way. In particular, since 2004 it has served as the top level ontology for the suite of orthogonal reference ontologies within the framework of the Open Biological and Biomedical Ontologies (OBO) Foundry. BFO is also very well documented, and many guidelines and training materials are available targeted to different audiences.

Examples of BFO-based engineering ontologies include the Computer Aided Engineering Modeling Language Ontology (CAEMLOnto) [18], the Product Service System Ontology (PSSO) [19], the Part-Focused Manufacturing Process Ontology (PMPO) [20], the ontology of the skill modeling for digital factories [21] the Innovative Capabilities of Additive Manufacturing Ontology (ICAM) [22], the Additive Manufacturing Ontology (AMO) [23], the Interoperability and Integration Framework (IoIF) [24], the DILECO OWL Ontology for the application of aircraft ground structure testing [25], the Physics-based Simulation Ontology [26], the Reference Ontology for the Maintenance Management Domain (ROMAIN) [27], the Supply Chain Reference Ontology [28], and the Process-Centric Systems Engineering Reference Ontology [29].

There is a proposal to adopt relevant modules from the Common Core Ontology $(\mathrm{CCO})^{2}$ as a mid-level for the IOF. The CCO was created by Ron Rudnicki and his collaborators at CUBRC, Inc. in Buffalo. Their goal is to provide a suite of ontologies and a rule-based method for extending these ontologies to represent the content of any data source whatsoever [30]. The CCO consists of ten mid-level ontologies that are extended from BFO. The Quality Ontology, one of the mid-level ontologies in the CCO, represents the attributes of agents, artifacts, and events.

Responding to a call for proposals from the Digital Manufacturing and Design Innovation Institute (DMDII) CUBRC and the University at Buffalo initiated the Coordinated Holistic Alignment of Manufacturing Process (CHAMP) project [31] with the goal of creating a flexible suite of interoperable generic public-domain ontologies covering the design, manufacturing, testing process, maintenance and tool domains. Through the CHAMP project, the Product Life Cycle Ontologies were created, and the FGMO 2.0, a revision of the FGMO [2] (named as FGMO 1.0 after this point onward) was developed as potential contribution to the IOF framework.

\footnotetext{
${ }^{2}$ See https://github.com/CommonCoreOntology/CommonCoreOntologies
} 


\subsection{The Product Life Cycle Ontologies}

The Product Life Cycle Ontologies ${ }^{3}$ are developed through the CHAMP project and funded by DMDII [32]. The Product Life Cycle Ontologies descend from BFO and CCO and are designed to assist in managing discordant manufacturing data sources that concern the whole life of a product in the market from design through manufacture, test, use, maintenance, and finally disposal for all phases. The ontologies include six mid-level ontologies, including a Manufacturing Process Ontology. In fact, the Additive Manufacturing Ontology (AMO) [22] is developed by extending the Manufacturing Process Ontology from The Product Life Cycle Ontologies.

\section{FGMO 1.0 Ontology Development}

The FGMO 1.0 may be divided into three levels: top-, mid-, and domain-level. The toplevel ontology terms are populated by BFO [33] [34]. The mid-level is modeled on the Ontology for Biomedical Investigations (OBI) [35]. Furini et al. [2] detail the FGMO 1.0, which covers FGM research in the area of components, manufacturing processes, material properties, and applications, focusing on the domain level to include low-level classes related to directly to FGM research [36] [37] [38].

BFO boosts the reuse of domain ontologies by serving as a standard top-level structure, allowing it to impose constraints on the classes and relations used by the midand lower-level ontologies. Its highest class, 'entity', includes everything in the domain of representation.

BFO distinguishes between two key, top-level entities: continuant and occurrent. A continuant is an entity that persists or maintains its self-identity through time [15]. The continuant class is divided into three subclasses: generically dependent continuant, independent continuant, and specifically dependent continuant. A generically dependent continuant is dependent on one or more independent continuants that serve as its bearer, but it can migrate from one bearer to another, for example through a process of copying. This class subsumes the IAO class information content entity, which includes documents, databases, requirements specifications, process specifications, and so forth, which are copied for example from one server to another. The material entity is the most important subclass of independent continuant; these are continuants that have some portion of matter as part. A specifically dependent continuant is an entity that relies on some specific independent continuant or continuants to exist. Subclasses of specifically dependent continuant include quality and realizable entity. Qualities such as a particular color, or a particular height, do not require any processes to be realized. They simply manifest themselves wherever they exist. By contrast, realizable entities, such as a role or function, are defined by the fact that they can be realized in some process. Examples of roles include social roles, such as the role of the employer and the role of the student. Examples of functions contain the function of your heart to pump blood. FGM classes for

${ }^{3}$ See https://github.com/NCOR-US/CHAMP 
material composition, material attributes, and the material entities that participate in given processes are subsumed under BFO's continuant classes.

The BFO occurrent class includes processes that happen, or occur in time, as well as, process boundaries, temporal and spatiotemporal regions [15]. Here, process is the most crucial subtype; every process requires that at least one material entity participates in the process and that the material entity exists at the time of the process. The FGM class manufacturing process is thus a subclass of the BFO class: process.

Process and material entity classes collected from BFO form the mid-level and the domain-level of the FGMO. This mid-level ontology is created by borrowing classes from the Ontology for Biomedical Investigations (OBI). OBI is an ontology that is intended to describe all aspects of investigations in the biological and medical domains [39]. OBI represents information and material entities that participate in the investigation processes, as well as roles and functions.

At the domain level, FGMO 1.0 includes the basic function classes related to FGMs. Our work here concentrates on improving the FGMO structure at the domain level, above all, by adding more classes relating to the FGM types, applications, and manufacturing processes and providing a reorganization of the FGMO structure to better support queries addressed against the data that are of interest to FGM researchers.

\section{FGMO 2.0}

\subsection{The enriched FGMO structure}

FGMO 2.0 retains the top-down approach adopted in FGMO 1.0, which means that ontology development proceeds through downward population from BFO and BFOconformant domain ontologies. However, we were able to draw on the PLC ontologies to redefine classes in FGMO 1.0 in order to ensure adequate alignment with features of the manufacturing domain. Figure 1 shows the import structure of these ontologies in FGMO 2.0 .

The new version of MatOnto mentioned in Section 2.2 is currently being reconstructed within the IOF framework. Since this new MatOnto is still a work in progress, we import only selected classes from this framework into the FGMO 2.0.

On the other hand, the Manufacturing Process Ontology as introduced in Section 2.4 is imported in full, and this means that the classes from OBI used in FGMO 1.0 are no longer needed. 


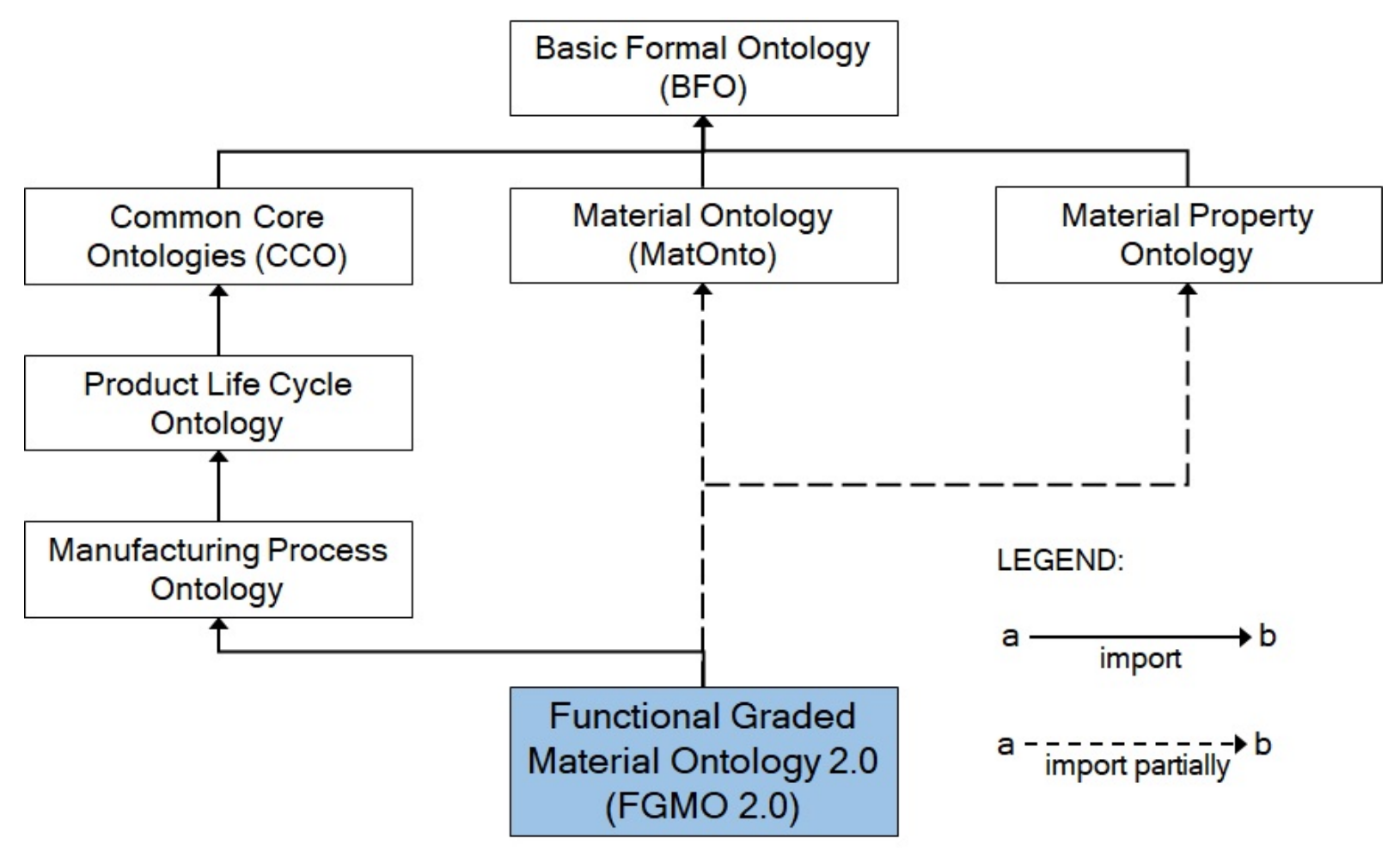

\subsection{Composition material hierarchy of FGMO}

The composition substances used by FGM include metal, ceramic as well as combinations of these with many other types of materials. FGMO 1.0 contains no definitions or elucidations for most material classes used in the ontology, and some of the definitions that are included are of poor quality. In addition, certain classes of materials were inappropriately classified, for instance, in that portions of material were classified as children of BFO:object. BFO distinguishes three main subtypes of material entity: object, fiat object part, and object aggregate. However, it does not assert that all material entities must fall under one of these headings. Thus, FGMO 2.0 classifies portions of material as children of material entity, thereby including for instance plasmas, liquids, filaments, or clouds of gas.

FGMs are a new type of material, engineered to fulfill specific functions. We believe there is a need for a material ontology that represents general classes of industrial materials as these are used as inputs to the processes specific to FGMs, and we anticipate that MatOnto will fill this role and will serve as an important mid-level ontology not just for the FGMO but for a range of other ontologies within the IOF framework and beyond. In the latest version of MatOnto, the material taxonomy is modified and now includes the improved definitions. Figure 2 illustrates how we have changed the range of subclasses of material entity in our update. 


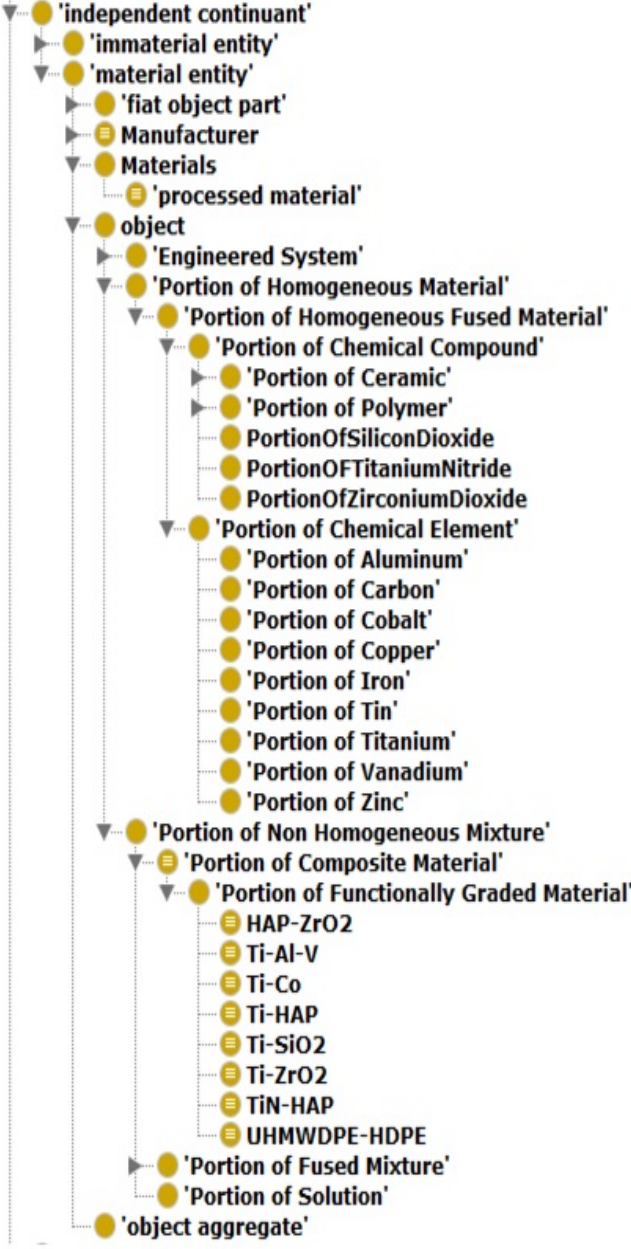

(a)

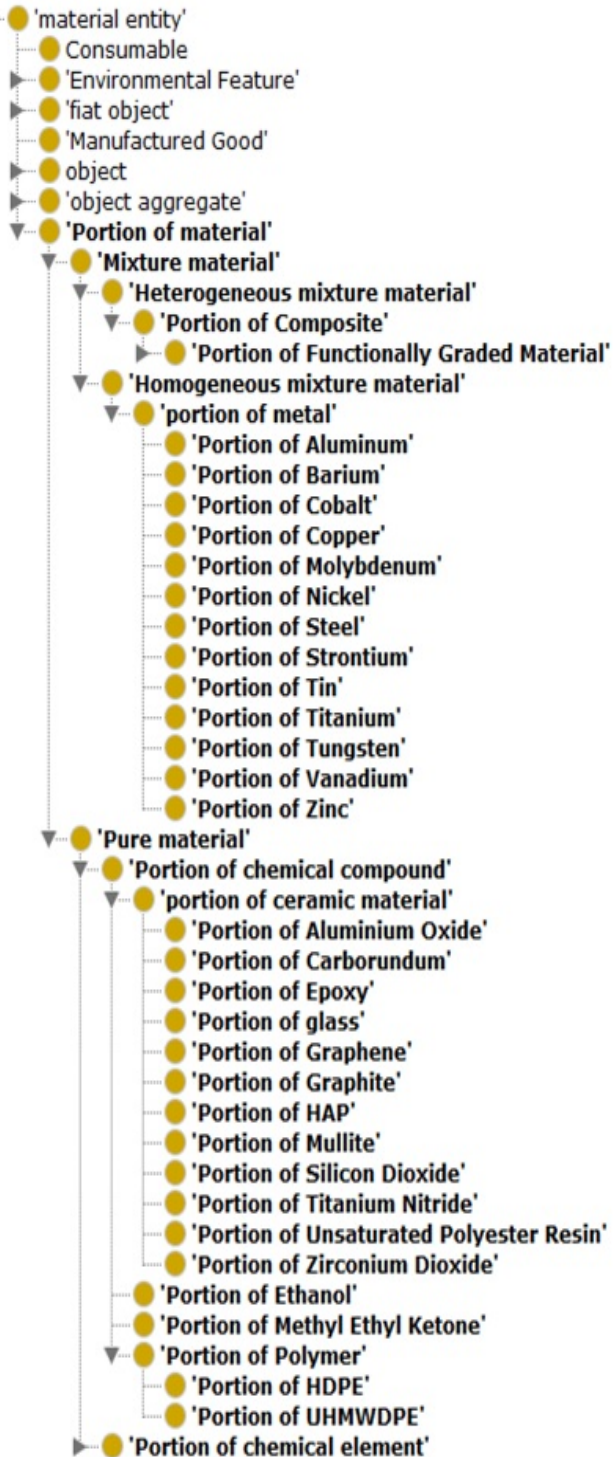

(b)

\subsection{Material properties of FGM}

Material selection is an important step in engineering design in which the reliability of the designed entity in meeting the product performance goals is determined. Material selection for a given product begins with a consideration of the properties and costs of candidate materials. First, materials with properties that satisfy the functional requirements and operating conditions of the product being designed are selected. Hardness and strength are examples of material properties. A functionally graded material is a product of the combination of different types of materials, so it too is subject to a material selection process. The coverage of material properties in FGMO 2.0 allows 
many different sorts of material properties of interest to be accounted for. In the Material Property Ontology, they are categorized under the BFO classes quality and disposition, which are defined as follows [33]:

- Quality is a specifically dependent continuant that inheres in an entity. This includes, for example, the mass of an entity.

- Disposition is a realizable entity that is associated with certain kinds of processes or activities in which the disposition can be realized. This includes, for example, the fragility of a crystal glass that can be realized by breaking, following an impact from falling to the floor.

A snapshot of the classes from the Material Property Ontology is shown in Figure 3.

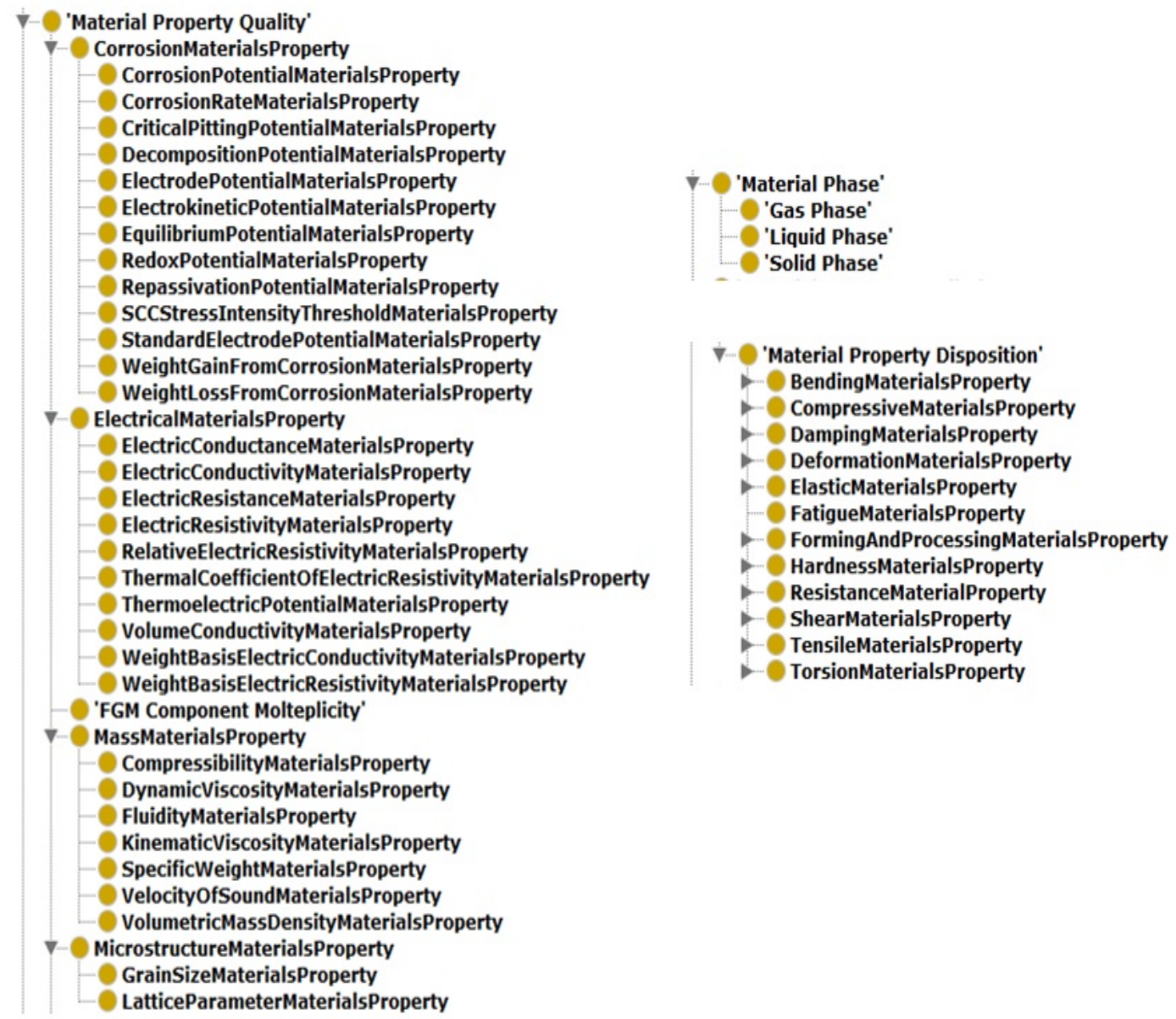

\subsection{The FGM manufacturing process}

FGMs are the advanced materials in the family of engineering composites that are made of two or more constituent materials with continuous and smoothly varying distribution [40]. The properties of FGM depend not only on the selected materials but also on the FGM fabrication process. 
In FGMO 1.0, FGM fabrication processes were represented using the classes: Manufacturing Process with Assembly Process, Composite Material Process, Joining Process, Material Additive Process, and Material Removal Process (see Figure 4). In version 2.0, however, the realm of material additive processes is now significantly expanded to account for the sorts of cases described in [41] [42] [43] [44]. These include the thermal spray process that is used mostly for the surface treatment of materials to achieve superalloys with high levels of resistance to erosion and other attributes useful in working tools, electromagnetic interfaces, and chemical and thermal barrier coatings [45]. Electrodeposition is another process where the composition gradient can be controlled by varying electrochemical factors and electrolytic solution [46]. And the expansion for the FGM manufacturing process also includes the additive manufacturing processes where the stereolithography-based, laser-based, material-jetting-based processes and surface reaction processes are added [47] [48] [49] [50] [51] [52] [53]. Additional additive manufacturing processes that have been added include selective laser melting, fused deposition modeling, laser engineered net shaping, and electron beam melting processes. In this way, the Manufacturing Process Ontology is being expanded to complete the representation of processes related to FGMs in FGMO 2.0.

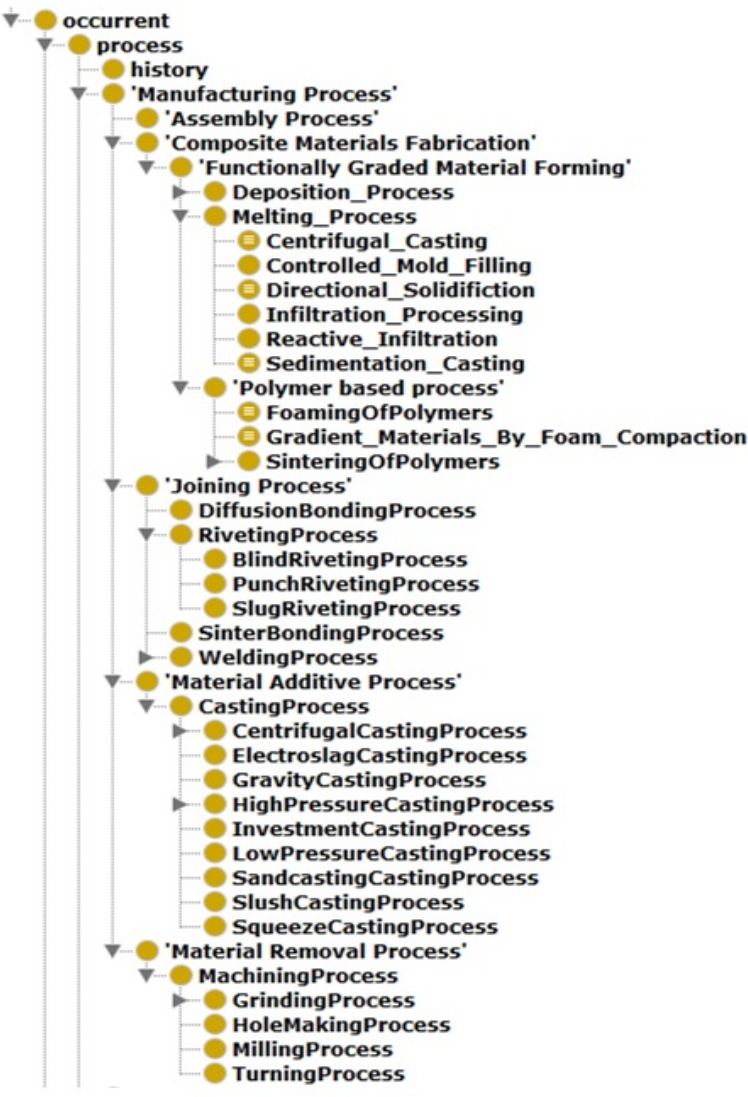

(a)

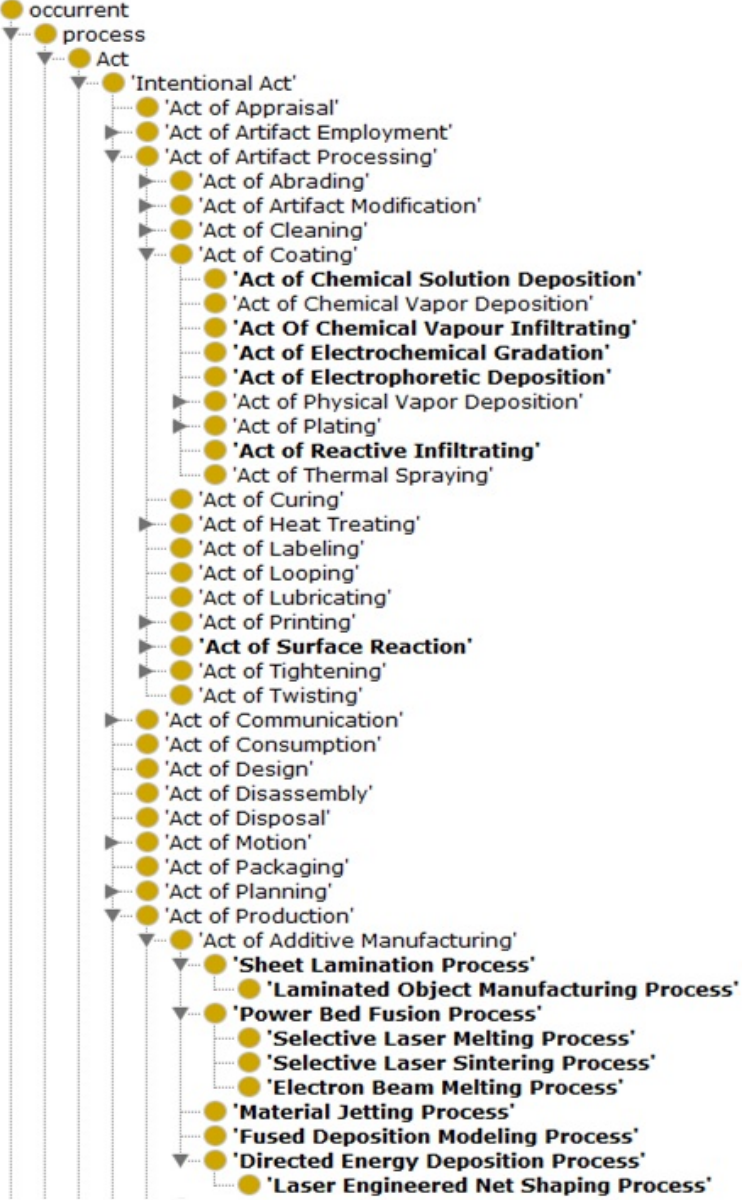

(b) 


\subsection{Applications of the FGMO}

FGM has been used in many applications due to its capability to possess multiple functional characteristics simultaneously. Some of the applications are in the area of aerospace, automobile, defense, energy, medical, nuclear science, and optoelectronics [54] [55].

To preserve its utility, the FGMO must keep pace with the large number of new applications that FGMs are making possible. FGMO 1.0 supported primary applications of FGMS in the domains of bone implants and aerospace engineering. More applications are included in the FGMO 2.0 and are represented in the forms of artifacts rather than application. CCO provides a good representation of various types of artifact. New classes, such as nuclear reactor component, chemical plant, aircraft engine, bullet-proof vests, tennis rackets, fire retardant doors, and so on have been added to the artifact classes in FGMO 2.0 (see Figure 5).

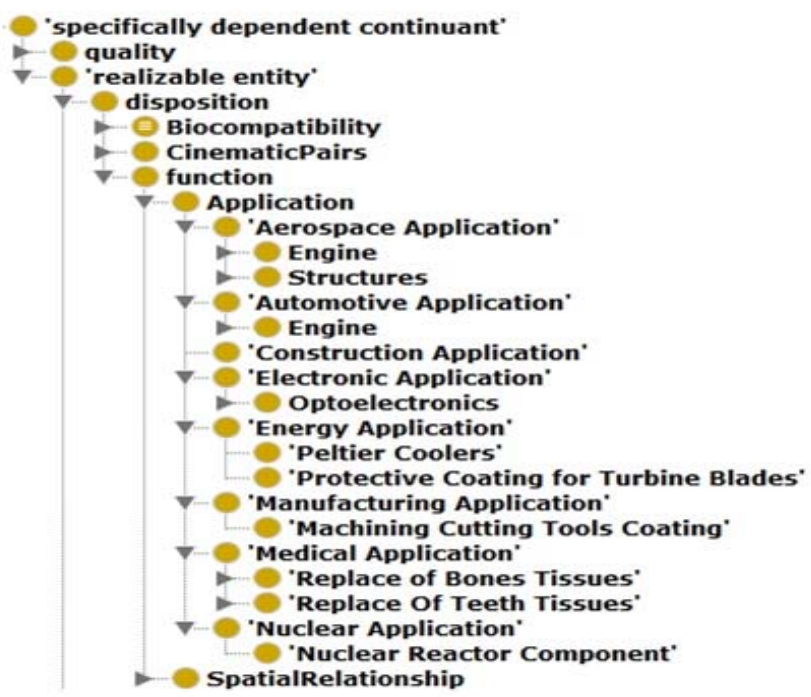

(a)

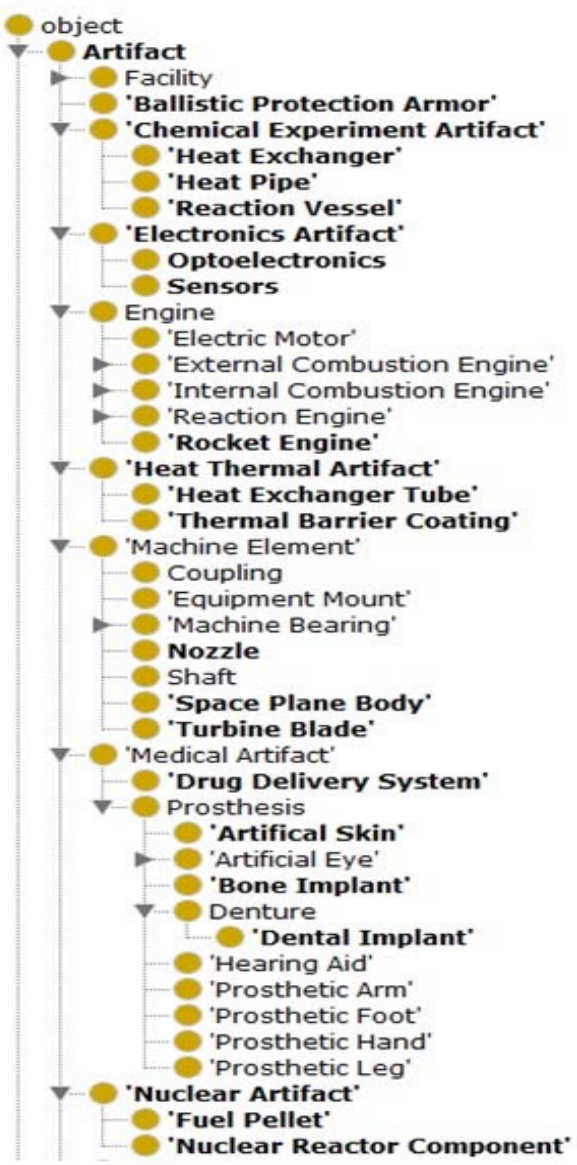

(b) 


\subsection{Relations}

Whereas FGMO 1.0 drew its relations mainly from the Relation Ontology (RO), FGMO 2.0 adopts several further relations from CCO, as illustrated in Table 1. Figure 6 provides an overview of the entities in the FGMO 2.0 and their relationships.

Table 1. Relations in FMGO 2.0 from RO and CCO.

\begin{tabular}{|l|l|}
\hline \multicolumn{1}{|c|}{ RO } & \multicolumn{1}{c|}{ CCO } \\
\hline $\begin{array}{l}\text { has_part } \\
\text { has_participant } \\
\text { part_of } \\
\text { participates_in }\end{array}$ & $\begin{array}{l}\text { is input of } \\
\text { is output of } \\
\text { process part of } \\
\text { bearer of } \\
\text { inheres in } \\
\text { is about } \\
\text { describes } \\
\text { designates } \\
\text { prescribes }\end{array}$ \\
\hline
\end{tabular}




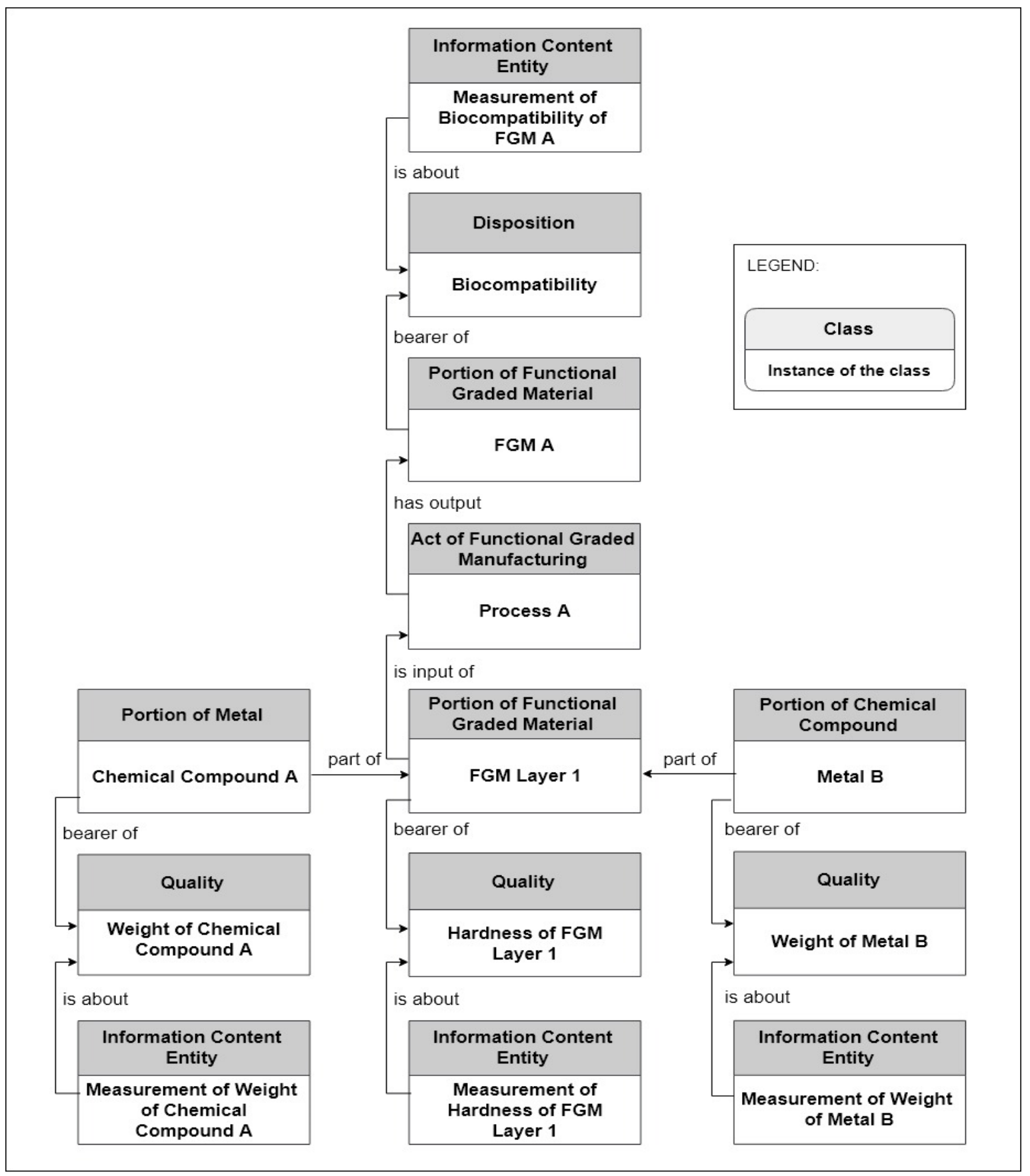

In what follows, we apply three samples of class expression in FGMO 2.0 to identify FGMs according to the manufacturing processes and applications involved. Class expressions are written in Manchester Syntax.

1. Portion of Material and (has_part some 'Portion of Polymer') and (isAppliedTo ' some Nozzle') and (is_output_of some 'Act of Laser Beam Welding') FGM type retrieved: Aluminum-Titanium 
2. Portion of Material and (has_part some 'Portion of Polymer') and (isAppliedTo some 'Turbine Blades') and (is_output_of some 'Act of Chemical Vapour Deposition')

FGM type retrieved: Carbon-Carborundum

3. Portion of Material and (has_part some 'Portion of Polymer') and (is_output_of some 'Act of Electrophoretic Deposition') FGM type retrieved: Aluminum Oxide-Zirconium Dioxide

\subsection{System architecture for FGMO 2.0 implementation}

Figure 7 shows the proposed system architecture for the FGMO 2.0 implementation in

digital manufacturing. Data related to FGM from various sources are to be managed in a data management system. In the system, the categories of the data are mapped as instances to the classes in the ontology, with the data also providing string values related to instances. The ontology with the tagged data, constitutes a semantic data model of the FGMO 2.0. The semantic data model accommodates derived information in a structural database specification that describes the classifications and groupings of the entities with the tagged data, and fundamental interconnections between them. The queries provided by the user will formulate the answers from the semantic data model.

\section{Examples of Semantic Queries Concerning FGM Materials}

In this section, three examples of FGM applications are used to demonstrate the practical usage of FGMO (examples are based on [38] [56] [57] [58] [59]). 


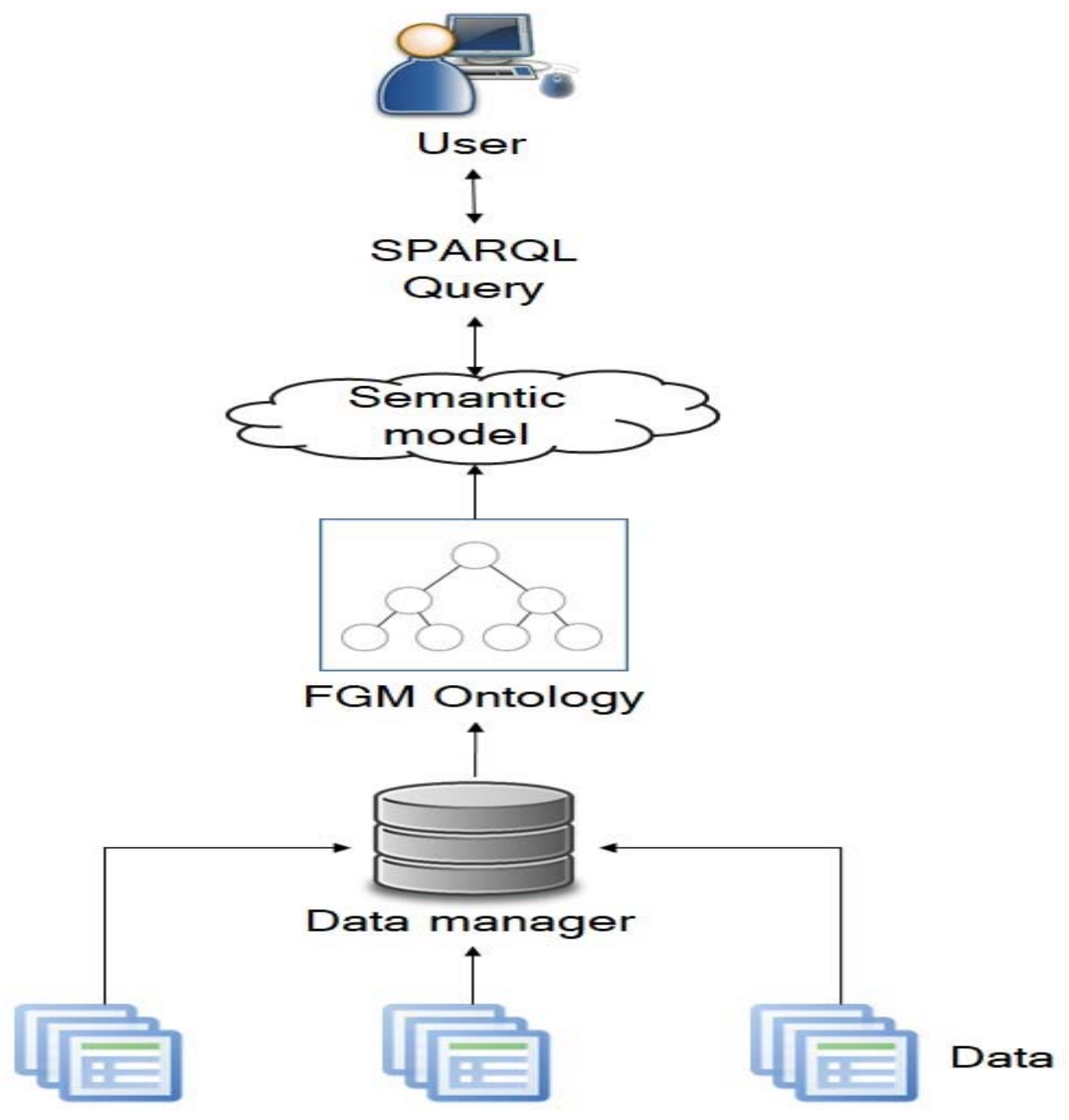

\subsection{Example 1: FGM and its properties and applications}

The first example starts with general information on the type of FGM and its applications, manufacturing processes, and material property [38] [56] [57]. The data involved are presented in Table 2. 
Table 2. FGM and its requirement, process, and application.

\begin{tabular}{|l|l|l|l|}
\hline FGM & Material Requirement & Manufacturing Process & Application \\
\hline $\begin{array}{l}\text { Carbon - } \\
\text { Carborundum }\end{array}$ & $\begin{array}{l}\text { High thermal } \\
\text { resistance Low } \\
\text { biocompatibility }\end{array}$ & $\begin{array}{l}\text { Chemical Vapor } \\
\text { Deposition }\end{array}$ & Protective Coating \\
\hline $\begin{array}{l}\text { Alumina } \\
\text { Zirconia }\end{array}$ & $\begin{array}{l}\text { High thermal } \\
\text { resistance High } \\
\text { biocompatibility }\end{array}$ & $\begin{array}{l}\text { Electrophoretic } \\
\text { Deposition }\end{array}$ & Thermal Barrier \\
\hline Nickel - Alumina & $\begin{array}{l}\text { High thermal } \\
\text { resistance } \\
\text { High compressive } \\
\text { strength }\end{array}$ & Powder Metallurgy & Body Armor \\
\hline
\end{tabular}

The following is a list of queries in natural language that we selected to test whether the FGMO can provide the needed inferred information:

a) Which FGM has a thermal resistance property that needs to be measured?

b) Which FGM has thermal resistance and compressive strength properties that need to be measured?

c) Which FGM has a low biocompatibility property?

Query 1a: Which FGM has a thermal resistance property that needs to be measured?

We used the following simple SPARQL query to find the FGM that it's thermal resistance property need to be measured. Figure 8a shows the result of the query.

SELECT ?FGM

WHERE \{

?FGM rdf:type matpro:PortionofMaterial.

?FGM cco:bearer_of ?b.

?b rdf:type fgmo:ThermalResistanceMaterialProperty.\}

Query 1b: Which FGM has thermal resistance and compressive strength properties that need to be measured?

The following SPARQL query was used to find FGMs that bear both a thermal resistance property and a compressive strength property. The results are shown in Figure 8b. 
SELECT ?FGM

WHERE \{

?FGM rdf:type matpro:PortionofMaterial.

?FGM cco:bearer_of ?b.

?b rdf:type fgmo:ThermalResistanceMaterialProperty .

?FGM cco:bearer_of ?c.

?c rdf:type matonto:CompressivestrengthMaterialsProperty\}

Query 1c: Which FGM has a low biocompatibility property?

The following SPARQL query that increases the number of constraints placed upon FGM selection by adding data was used to find the FGM that has a high biocompatibility property. The results are shown in Figure 8c.

SELECT ?FGM

WHERE \{

?FGM rdf:type matpro:PortionofMaterial.

?FGM cco:bearer_of ?b.

?b rdf:type fgmo:Biocompatibility.

?c cco:is_about ?b.

?c rdf:type cco:InformationContentEntity.

?c cco:inheres_in ?d.

?d rdf:type cco:InformationBearingEntity.

?d cco:has_text_value "Low biocompatibility".

\} 


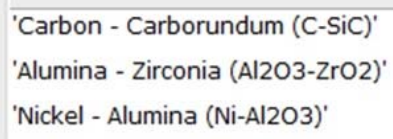

(a)

\section{'Nickel - Alumina (Ni-Al2O3)'}

(b)

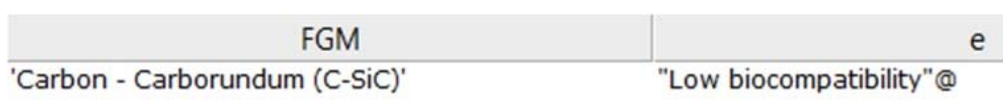

(c)

\section{FGM}

"Low biocompatibility"@

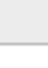

\subsection{Example 2: Density and compressive strength properties of Aluminum-}

Carborundum (Al-SiC)

This example concerns the measurement of the density and compressive strength of a portion of Aluminum-Carborundum (Al-SiC) FGM that is fabricated by powder metallurgy using different pressure and temperature settings. Besides the density and compressive strength measurement, the hardness of each layer in the FGM is also measured, where differences in the weight percentage of Aluminum and Carborundum are also varied. The relevant data are tabulated in Table 3 (from Kirmizi et al. [58]).

Table 3. Al-SiC fabrication and analysis data.

\begin{tabular}{|c|c|c|c|c|c|c|c|}
\hline FGM & Layer & $\begin{array}{c}\text { Composition } \\
(\%)\end{array}$ & $\begin{array}{c}\text { Hardnes } \\
\text { s (HB) }\end{array}$ & $\begin{array}{c}\text { Pressure } \\
(\mathrm{MPa})\end{array}$ & $\begin{array}{l}\text { Temp. } \\
\left({ }^{0} \mathrm{C}\right)\end{array}$ & $\begin{array}{l}\text { Density } \\
\left(\mathrm{g} / \mathrm{cm}^{3}\right)\end{array}$ & $\begin{array}{c}\text { Compressive } \\
\text { Strength } \\
(\mathrm{MPa})\end{array}$ \\
\hline \multirow{4}{*}{$\begin{array}{l}\mathrm{Al}- \\
\mathrm{SiC} 1\end{array}$} & 1 & $\begin{array}{l}15 \% \mathrm{SiC}+ \\
85 \% \mathrm{Al}\end{array}$ & 69.5 & \multirow{4}{*}{200} & \multirow{4}{*}{300} & \multirow{4}{*}{2.32} & \multirow{4}{*}{44.2} \\
\hline & 2 & $\begin{array}{l}30 \% \mathrm{SiC}+ \\
70 \% \mathrm{Al}\end{array}$ & 60.8 & & & & \\
\hline & 3 & $\begin{array}{l}45 \% \mathrm{SiC}+ \\
55 \% \mathrm{Al}\end{array}$ & 47.8 & & & & \\
\hline & 4 & $\begin{array}{l}60 \% \mathrm{SiC}+ \\
40 \% \mathrm{Al}\end{array}$ & 29.16 & & & & \\
\hline \multirow{3}{*}{$\begin{array}{l}\mathrm{Al}- \\
\mathrm{SiC} 2\end{array}$} & 1 & $\begin{array}{l}15 \% \mathrm{SiC}+ \\
85 \% \mathrm{Al}\end{array}$ & N/A & \multirow{3}{*}{300} & \multirow{3}{*}{400} & \multirow{3}{*}{2.83} & \multirow[b]{3}{*}{512.1} \\
\hline & 2 & $\begin{array}{l}30 \% \mathrm{SiC}+ \\
70 \% \mathrm{Al}\end{array}$ & N/A & & & & \\
\hline & 3 & $\begin{array}{l}45 \% \mathrm{SiC}+ \\
55 \% \mathrm{Al}\end{array}$ & N/A & & & & \\
\hline
\end{tabular}




\begin{tabular}{|c|c|c|c|c|c|c|c|}
\hline & 4 & $\begin{array}{l}60 \% \mathrm{SiC}+ \\
40 \% \mathrm{Al}\end{array}$ & N/A & & & & \\
\hline \multirow{4}{*}{$\begin{array}{l}\mathrm{Al}- \\
\mathrm{SiC} 3\end{array}$} & 1 & $\begin{array}{l}15 \% \mathrm{SiC}+ \\
85 \% \mathrm{Al}\end{array}$ & 89.9 & \multirow{4}{*}{400} & \multirow{4}{*}{500} & \multirow{4}{*}{2.94} & \multirow{4}{*}{704.5} \\
\hline & 2 & $\begin{array}{l}30 \% \mathrm{SiC}+ \\
70 \% \mathrm{Al}\end{array}$ & 112.33 & & & & \\
\hline & 3 & $\begin{array}{l}45 \% \mathrm{SiC}+ \\
55 \% \mathrm{Al}\end{array}$ & 153.33 & & & & \\
\hline & 4 & $\begin{array}{l}60 \% \mathrm{SiC}+ \\
40 \% \mathrm{Al}\end{array}$ & 193.66 & & & & \\
\hline
\end{tabular}

We provide three queries pertaining to these data.

Query 2a: What are the pressure and temperature values to be set for the powder metallurgy process to produce the highest density Al-SiC?

The following SPARQL query was used to determine the pressure and temperature values that are required for the process that produces Al-SiC with the highest density value. The results are shown in Figure 9a.

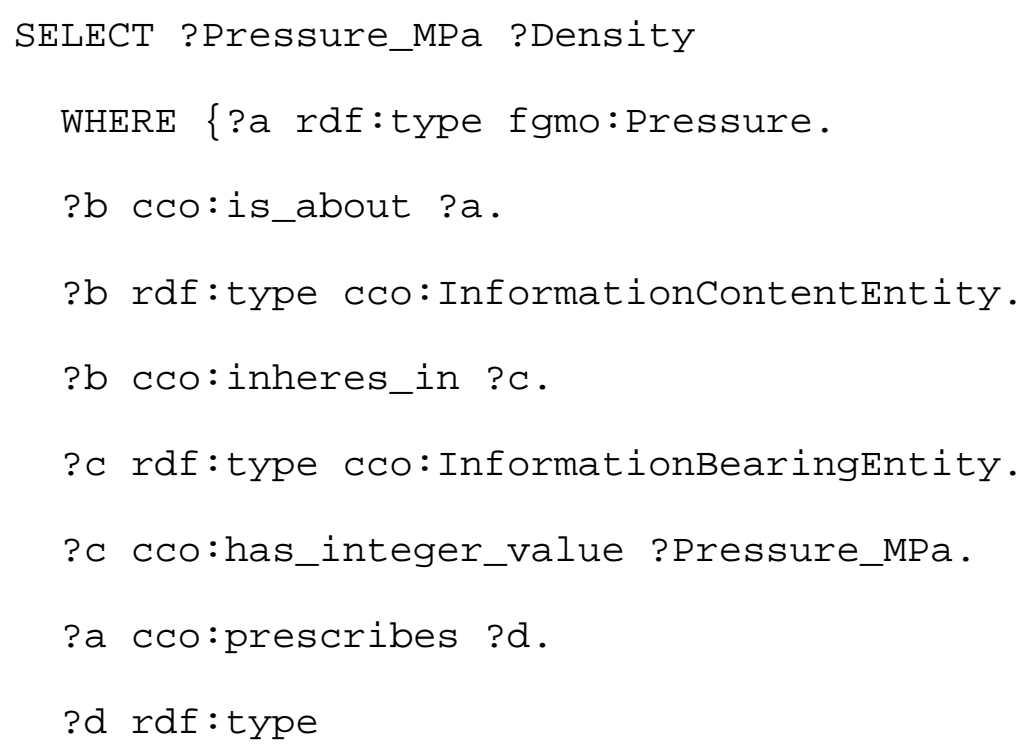


?g cco:is_about ?f.

?g rdf:type cco: InformationcontentEntity.

?g cco:inheres_in ?h.

?h rdf:type cco:InformationBearingEntity.

?h cco:has_decimal_value ?Density.\}

ORDER BY DESC(?i) LIMIT 1

Query 2b: What is the hardness for the Al-SiC that have a particular composition of Al and $\mathrm{SiC}$ ?

The following SPARQL query was used to find the hardness value for each layer of the FGM defined according to material percentage composition:

SELECT ?SiCWeightPercentage ?AlWeightPercentage

?Hardness_HB

WHERE $\{$ ? a rdf:type

fgmo:PortionofFunctionallyGradedMaterial.

?a cco:bearer_of ?b.

?b rdf:type cco:Hardness.

?c cco:is_about ?b.

?c rdf:type cco:InformationcontentEntity.

?c cco:inheres_in ?d.

?d cco:has_decimal_value ?Hardness_HB.

?e ro:part_of ?a.

?e rdf:type fgmo:PortionofCarborundum.

?e cco:bearer_of ?f.

?f rdf:type cco:Weight.

?g cco:is_about ?f.

?g rdf:type cco:InformationcontentEntity.

?g cco:inheres_in ?h.

?h rdf:type cco: InformationBearingEntity. 
?h cco:has_integer_value ?SiCWeightPercentage.

?i ro:part_of ?a.

?i rdf:type fgmo:Portionofaluminum.

?i cco:bearer_of ?j .

?j rdf:type cco:Weight.

?k cco:is_about ?j.

?k rdf:type cco:InformationContentEntity.

?k cco:inheres_in ?l.

?l rdf:type cco:InformationBearingEntity.

?l cco:has_integer_value ?AlWeightPercentage.\}

ORDER BY DESC(?Hardness_HB)

The results are shown in Figure 9b.

Query 2c: What are the pressure and temperature values to be set for the powder metallurgy process to produce the hardest $\mathrm{Al}-\mathrm{SiC}$ among the sample?

The following SPARQL query was used to find the pressure and temperature values required by the process that produces the $\mathrm{Al}-\mathrm{SiC}$ layer that has the highest hardness value. The results are shown in Figure 9c.

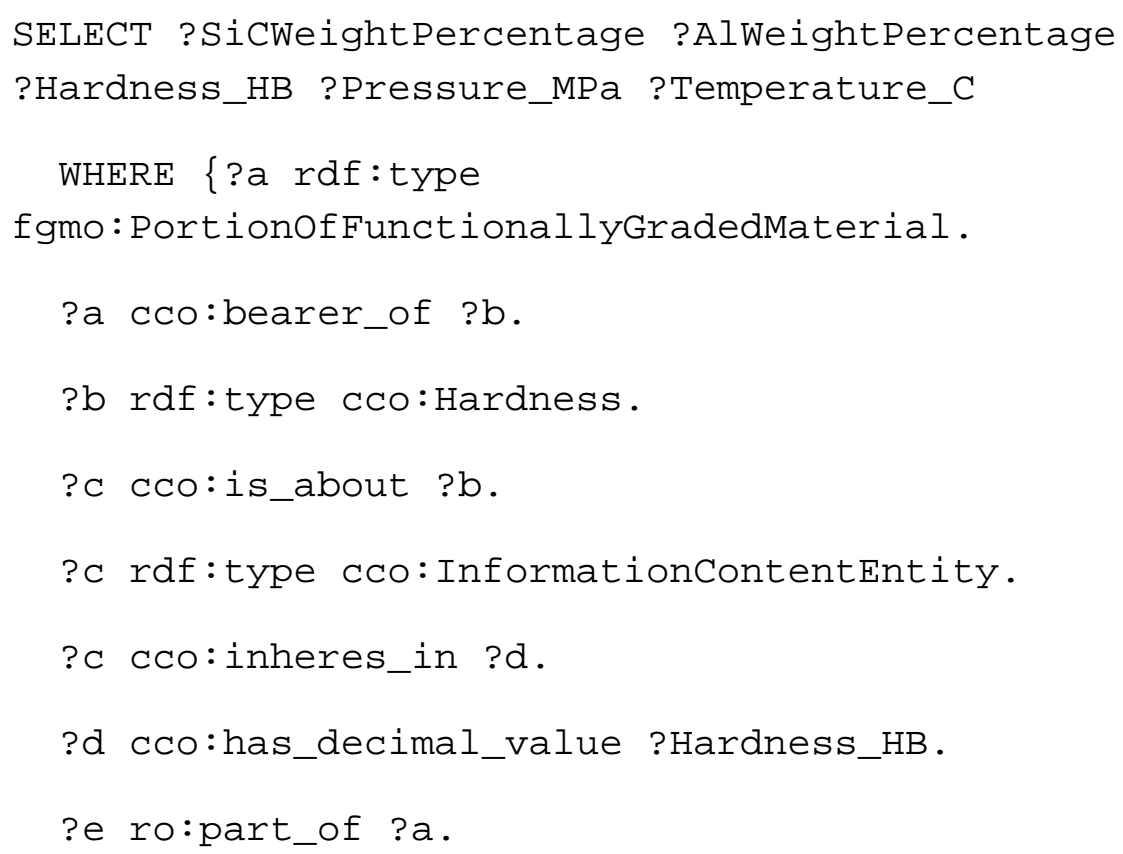


?e rdf:type fgmo:PortionofCarborundum.

?e cco:bearer_of ?f .

?f rdf:type cco:Weight.

?g cco:is_about ?f.

?g rdf:type cco:InformationContentEntity.

?g cco:inheres_in ?h.

?h rdf:type cco:InformationBearingEntity.

?h cco:has_integer_value ?SiCWeightPercentage.

?i ro:part_of ?a.

?i rdf:type fgmo:Portionofaluminum.

?i cco:bearer_of ?j.

?j rdf:type cco:Weight.

?k cco:is_about ?j.

?k rdf:type cco:InformationcontentEntity.

?k cco:inheres_in ?l.

?1 rdf:type cco:InformationBearingEntity.

?l cco:has_integer_value ?AlWeightPercentage.

?a ro:part_of ?m.

?m rdf:type fgmo:PortionofFunctionallyGradedMaterial.

?m cco:is_output_of ?n.

?n rdf: type

fgmo: ActofFunctionallyGradedMaterialManufacturing .

?o cco:prescribes ?n.

?o rdf:type fgmo:Pressure.

?p cco:is_about ?o.

?p rdf:type cco:InformationcontentEntity.

?p cco:inheres_in ?q. 
?q rdf:type cco:InformationBearingEntity.

?q cco:has_integer_value ?Pressure_MPa.

?r cco:prescribes ?n.

?r rdf:type fgmo:Temperature.

?s cco:is_about ?r.

?s rdf:type cco:InformationcontentEntity.

?s cco:inheres_in ?t.

?t rdf:type cco:InformationBearingEntity.

?t cco:has_integer_value ?Temperature_c.

FILTER (?Hardness_HB=193.66)\}

This query is more complicated than queries $2 \mathrm{a}$ and $2 \mathrm{~b}$. Specifically, it employs the FILTER statement in applying one of the values obtained from Query $2 b$ to find the answer from Query 2b.

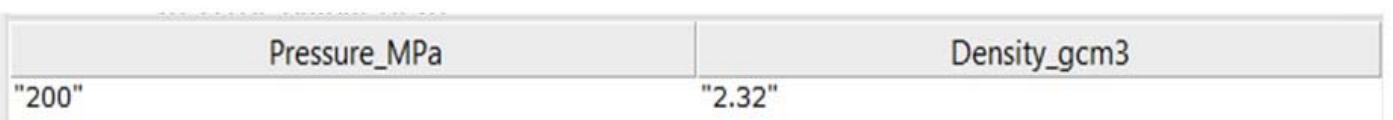

(a)

\begin{tabular}{|c|c|c|}
\hline SiCWeightPercentage & AlWeightPercentage & Hardness_HB \\
\hline "60" & "40" & "193.66" \\
\hline "45" & "55" & "153.33" \\
\hline "30" & "70" & "112.38" \\
\hline "15" & "85" & "89.9" \\
\hline "15" & "85" & "69.5" \\
\hline "30" & "70" & "60.8" \\
\hline "45" & "55" & "47.8" \\
\hline "60" & "40" & "29.16" \\
\hline
\end{tabular}

(b)

\begin{tabular}{|c|c|c|c|c|}
\hline SiCWeightPercentage & AlWeightPercentage & Hardness_HB & Pressure_MPa & Temperature_C \\
\hline 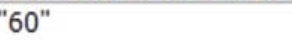 & "40" & "193.66" & "400" & "500" \\
\hline
\end{tabular}

(c) 


\subsection{Example 3: FGM types according to the additive manufacturing process type}

This example concerns the ability of additive manufacturing processes to produce a FGM from the different kinds of base materials. Table 4a shows the three different types of base material that are suitable to be used for different types of additive manufacturing processes in producing the FGM. As has been mentioned in Section 4.5, FGM has been used in many applications, and one of them is in orthopedic implants. Suitable metallic biomaterials have been developed to suit the numerous functions of such implants [59]. We focus in Table $4 \mathrm{~b}$ on the comparison of three different additive manufacturing processes used to produce metallic-based FGMs from the metal base material.

Table 4. FGM with the additive manufacturing processes.

(a)

\begin{tabular}{|c|c|c|}
\hline Base material type & Material name & Additive manufacturing process \\
\hline Metal-based & $\begin{array}{c}\text { Titanium, } \\
\text { Titanium alloy, } \\
\text { Stainless steel, } \\
\text { Cobalt chromium } \\
\text { alloy }\end{array}$ & $\begin{array}{c}\text { Laser- engineered net shaping, } \\
\text { Selective laser melting, } \\
\text { Electron beam melting }\end{array}$ \\
\hline Ceramic-based & $\begin{array}{c}\text { Alumina, Zircon } \\
\text { dioxide, } \\
\text { Hydroxyapatite } \\
\text { (HAP) }\end{array}$ & Selective laser sintering \\
\hline Polymer-based & $\begin{array}{c}\text { Polyproylene } \\
\text { polymer, } \\
\text { Tricalcium } \\
\text { phospate }\end{array}$ & Fused deposition modeling \\
\hline & & \\
\hline
\end{tabular}

(b)

\begin{tabular}{|c|c|c|c|}
\hline Material & Material form & $\begin{array}{c}\text { Additive manufacturing } \\
\text { process }\end{array}$ & FGM quality \\
\hline Powder & Selective laser melting & $\begin{array}{c}\text { High tensile } \\
\text { strength, } \\
\text { Good } \\
\text { accuracy }\end{array}$ \\
\hline
\end{tabular}




\begin{tabular}{|c|c|c|c|}
\cline { 2 - 4 } Titanium alloy & Powder & Electron beam melting & $\begin{array}{c}\text { Good } \\
\text { accura } \\
\text { cy, } \\
\text { High } \\
\text { density }\end{array}$ \\
\cline { 2 - 4 } & & $\begin{array}{c}\text { Laser-engineered net } \\
\text { shaping }\end{array}$ \\
Powder or wire & Low \\
& & & accuracy \\
\hline
\end{tabular}

We provide two queries for this example.

Query 3a: Which additive manufacturing processes can be used to produce metallicbased FGM?

The following SPARQL query was used. Results are shown in Figure 10a, where there are three additive manufacturing processes that can be applied to the metal base material.

SELECT ?Material ?AdditiveManufacturingProcess

WHERE \{

?a rdf:type matpro:Portionofmetal.

?a cco:has_text_value ?Material.

?a cco:is_input_of ?b.

?b rdf:type mpo:ActofAdditiveManufacturing.

?b cco:has_text_value ?AdditiveManufacturingProcess.\}

The goal of the next query is to select the additive manufacturing process that can produce FGM with some required characteristic.

Query 3b: Which additive manufacturing process can produce good accuracy FGM with the highest tensile strength?

The following SPARQL query was used. Results are shown in Figure 10b where selective laser melting (SLM) is the best candidate that can produce the required output.

SELECT ?Material ?AdditiveManufacturingProcess ?FGM ?f ?i

WHERE \{

?a rdf:type matpro:Portionofmetal. 


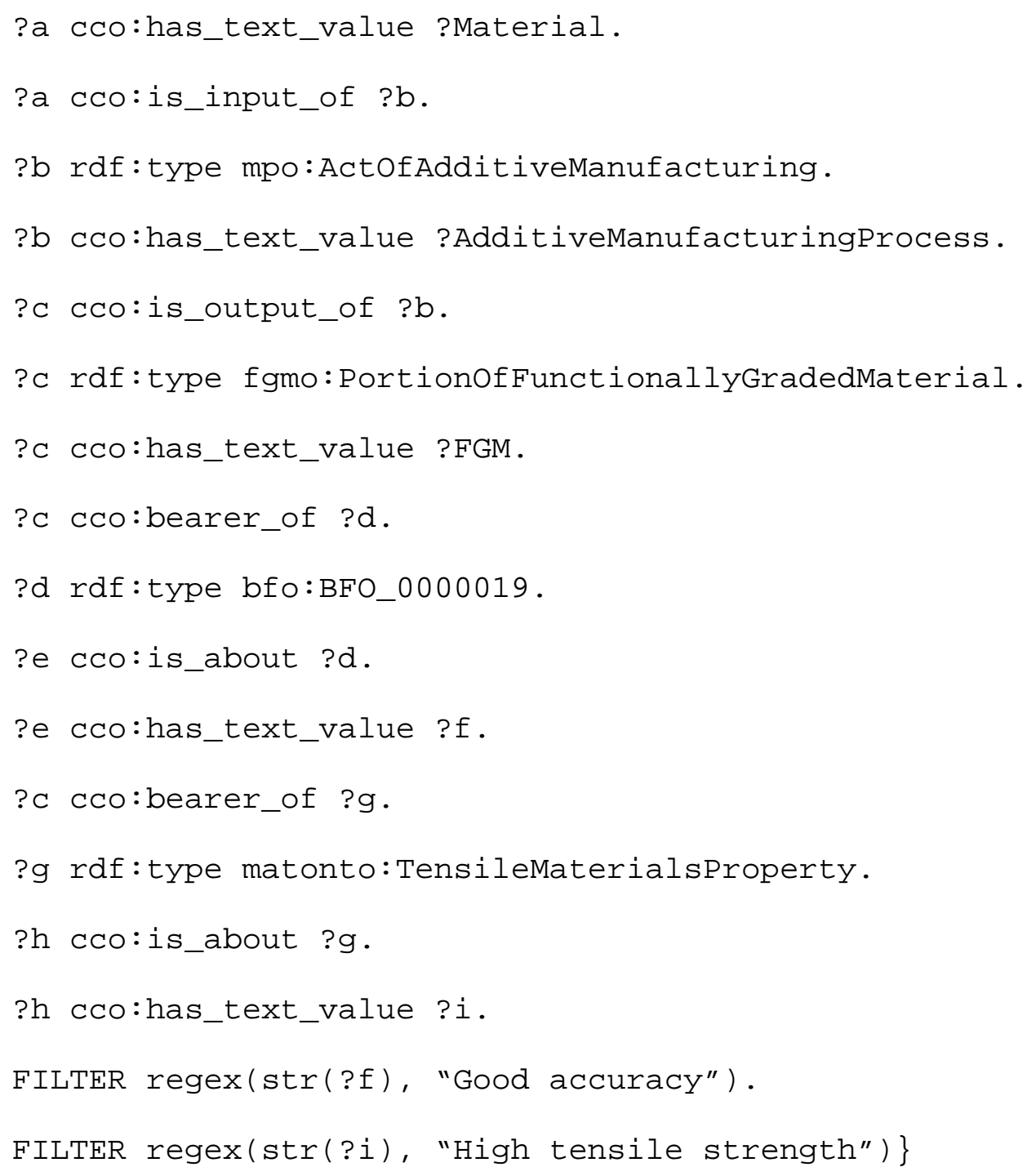

\begin{tabular}{|c|c|}
\hline Material & AdditiveManufacturingProcess \\
\hline "Titanium alloy"@ "Selective Laser Melting"@ \\
"Titanium alloy"@ "Electron Beam Melting"@ \\
"Titanium alloy"@ "Laser engineered net shaping"@
\end{tabular}

(a)

\begin{tabular}{|c|c|c|c|c|}
\hline Material & AdditiveManufacturingProcess & FGM & $f$ & i \\
\hline
\end{tabular}

(b)

Further queries can be conducted to identify process parameters for the additive manufacturing process, such as laser power and scanning speed. Here, relations similar to 
those used in Query 2c can be applied, since the process parameters are treated as the information content entity that prescribes the additive manufacturing process.

As these examples make clear, FGMO may be used to facilitate the discovery of useful information for those working in the FGM and related manufacturing domain. FGMO 2.0 is showing healthy signs of development that will be helpful to researchers. The FGMO has increased the number of FGM manufacturing application classes from twenty-nine to sixty-four and material classes from twenty-seven to fifty-two. In addition, the number of process classes has increased from twenty-seven to seventy-nine.

\section{Conclusion}

As data proliferates in the domain of FGMs and the way they are manufactured, ontologies are going to play a critical role in facilitating data integration across multiple sources of heterogeneous data. The FGMO attempts to address this need for integration and interoperability by providing a highly general representation of the domain. This will provide a single target to which highly diverse information may be mapped, thereby enabling new sorts of complex queries against a growing body of data. We have described here our work updating the FGMO 1.0 and extending its classes, relations, imported ontologies, and axioms as well as modifying and enriching definitions. We believe that FGMO will be a useful tool for researchers who need to retrieve and reason with information about FGMs in the digital manufacturing domain. In the future, we will not only continue to enrich the ontology but also integrate it with other ontologies designed for applications in industrial and manufacturing engineering within the framework of the Industry Ontology Foundry.

\section{References}

[1] Miyamoto, Yoshinari, WA Kaysser, BH Rabin, Akira Kawasaki, and Reneé G Ford. 2013. Functionally graded materials: design, processing and applications. Vol. 5: Springer Science \& Business Media.

[2] Furini, Francesco, Rahul Rai, Barry Smith, Georgio Colombo, and Venkat Krovi. 2016. "Development of a manufacturing ontology for functionally graded materials." ASME 2016 International Design Engineering Technical Conferences and Computers and Information in Engineering Conference.

[3] Rubin, Daniel L, Suzanna E Lewis, Chris J Mungall, Sima Misra, Monte Westerfield, Michael Ashburner, Ida Sim, Christopher G Chute, Margaret-Anne Storey, and Barry Smith. 2006. "National Center for Biomedical Ontology: advancing biomedicine through structured organization of scientific knowledge." Omics: a journal of integrative biology 10 (2):185-198.

[4] Smith, Barry. 2004. "Beyond concepts: ontology as reality representation." Proceedings of the third international conference on formal ontology in information systems (FOIS 2004):73-84. 
[5] Lemaignan, Severin, Ali Siadat, J-Y Dantan, and Anatoli Semenenko. 2006.

"MASON: A proposal for an ontology of manufacturing domain." IEEE Workshop on

Distributed Intelligent Systems: Collective Intelligence and Its Applications

(DIS'06):195-200.

[6] Mazzola, Luca, Patrick Kapahnke, Marko Vujic, and Matthias Klusch. 2016. "CDM-

Core: A Manufacturing Domain Ontology in OWL2 for Production and Maintenance."

Proceedings of the 8th International Joint Conference on Knowledge Discovery,

Knowledge Engineering and Knowledge Management (IC3K 2016) - Volume 2:

KEOD:136-143.

[7] Chang, Xiaomeng, Rahul Rai, and Janis Terpenny. 2010. "Development and utilization of ontologies in design for manufacturing." Journal of Mechanical Design 132 (2):021009.

[8] Ameri, Farhad, Colin Urbanovsky, and Christian McArthur. 2012. "A systematic approach to developing ontologies for manufacturing service modeling." Proceedings of the workshop on ontology and semantic web for manufacturing: page 14.

[9] Usman, Zahid, Robert Ian Marr Young, Nitishal Chungoora, Claire Palmer, Keith Case, and Jenny Harding. 2011. "A manufacturing core concepts ontology for product lifecycle interoperability.” International IFIP Working Conference on Enterprise Interoperability: 5-18.

[10] Garetti, Marco, and Luca Fumagalli. 2012. "P-PSO ontology for manufacturing systems." IFAC Proceedings Volumes 45 (6):449-456.

[11] Zhang, Xiaoming, Dongyu Pan, Chongchong Zhao, and Kai Li. 2016. "MMOY: Towards deriving a metallic materials ontology from Yago." Advanced Engineering Informatics 30 (4):687-702.

[12] Cheung, Kwok, John Drennan, and Jane Hunter. 2008. "Towards an Ontology for Data-driven Discovery of New Materials." AAAI Spring Symposium: Semantic Scientific Knowledge Integration: 9-14.

[13] Wallace, Evan, Dimitris Kiritsis, Barry Smith, and Chris Will. 2018. "The Industrial Ontologies Foundry Proof-of-Concept Project." In IFIP International Conference on Advances in Production Management Systems, 402-409. Springer.

[14] Rudnicky, Ron, and CUBRC. 2016. "WHITE PAPER Best Practices of Ontology Development." CUBRC.

https:/github.com/CommonCoreOntology/CommonCoreOntologies/tree/versions/docum entation.

[15] Arp, Robert, Barry Smith, and Andrew D Spear. 2015. Building ontologies with Basic Formal Ontology: Mit Press.

[16] Mascardi, Viviana, Valentina Cordì, and Paolo Rosso. 2008. "A comparison of upper ontologies (technical report disi-tr-06-21)." Dipartimento di Informatica e Scienze dell'Informazione (DISI), Università degli Studi di Genova.

[17] Borgo, Stefano, and Claudio Masolo. 2009. "Foundational choices in DOLCE." In Handbook on ontologies, 361-381. Springer. 
[18] Suwelack, Stefan, Markus Stoll, Annika Meyer, Steffen Slavetinsky, Manuel Serf, Nikola Bursac, Albert Albers, Rolf Bendl, Rüdiger Dillmann, and Stefanie Speidel. 2016. "Towards Cognitive Computer Aided Engineering." Proceedings of the International Association for the Engineering Modeling, Analysis and Simulation Community. [19] Correia, Ana, Dragan Stokic, Rebecca Siafaka, and Sebastian Scholze. 2017. "Ontology for colaborative development of product service systems based on basic formal ontology." International Conference on Engineering, Technology and Innovation (ICE/ITMC): 1173-1180.

[20] Mesmer, Lucas, and Andrew Olewnik. 2018. "Enabling supplier discovery through a part-focused manufacturing process ontology.” International Journal of Computer Integrated Manufacturing 31 (1):87-100..

[21] Arena, Damiano, Farhad Ameri, and Dimitris Kiritsis. 2018. "Skill Modelling for Digital Factories." In Advances in Production Management Systems. Smart Manufacturing for Industry 4.0. APMS 2018. IFIP Advances in Information and Communication Technology, 318-326. Springer.

[22] Hagedorn, Thomas J, Sundar Krishnamurty, and Ian R Grosse. 2018. “A Knowledge-Based Method for Innovative Design for Additive Manufacturing Supported by Modular Ontologies." Journal of Computing and Information Science in Engineering 18 (2):1-12.

[23] Ali, Munira Mohd, Rahul Rai, J Neil Otte, and Barry Smith. 2019. "A product life cycle ontology for additive manufacturing." Computers in Industry 105:191-203.

[24] Bone, Mary, Mark Blackburn, Benjamin Kruse, John Dzielski, Thomas Hagedorn, and Ian Grosse. 2018. "Toward an Interoperability and Integration Framework to Enable Digital Thread." Systems 6 (4):46.

[25] Arena, D, M Oliva, I Eguia, C Del Valle, and D Kiritsis. 2019. "Semantic ModelDriven PLM Data Interoperability: An Application for Aircraft Ground Functional Testing with Eco-Design Criteria." In Advances in Production Management Systems. Smart Manufacturing for Industry 4.0. APMS 2018. Production Management for the Factory of the Future. IFIP International Conference on Advances in Production Management Systems, 299-306. Springer.

[26] Cheong, Hyunmin, and Adrian Butscher. 2019. "Physics-based simulation ontology: an ontology to support modelling and reuse of data for physics-based simulation." Journal of Engineering Design:1-33.

[27] Karray, Mohamed Hedi, Farhad Ameri, Melinda Hodkiewicz, and Thierry Louge. 2019. "ROMAIN: Towards a BFO compliant reference ontology for industrial maintenance." Applied Ontology 14 (2):155-177.

[28] Kulvatunyou, Boonserm, and Farhad Ameri. 2019. "A Supply Chain Reference Ontology Based on Basic Formal Ontology." ASME 2019 International Design Engineering Technical Conferences \& Computers and Information in Engineering Conference (IDETC/CIE). 
[29] Orellana, Douglas, and William Mandrick. 2019. "The Ontology of Systems Engineering: Towards a Computational Digital Engineering Semantic Framework." Procedia Computer Science 153:268-276.

[30] Rudnicky, Ron, and CUBRC. 2016. "Common Core Ontologies (CCO)." https://www.cubrc.org/index.php/data-science-and-information-fusion/ontology. [31] Otte, J. Neil, Ron Rudnicki, and Barry Smith. 2018. "Final Report for Coordinated Holistic Alignment of Manufacturing Process (CHAMP) Base Year." https://github.com/NCOR-US/CHAMP. [32] Otte, J. Neil, Dimitris Kiritsi, Munira Mohd Ali, Ruoyu Yang, Binbin Zhang, Ron Rudnicki, Rahul Rai, and Barry Smith. 2019. “An ontological approach to representing the product life cycle.” Applied Ontology 14 (2):179-197.

[33] Arp, Robert, and Barry Smith. 2008. "Function, role and disposition in basic formal ontology." Proceedings of Bio-Ontologies Workshop, Intelligent Systems for Molecular Biology (ISMB), Toronto.

[34] Smith, Barry. 2012. "On classifying material entities in Basic Formal Ontology." In Interdisciplinary Ontology: Proceedings of the Third Interdisciplinary Ontology Meeting, 1-13. Keio University Press.

[35] Brinkman, Ryan R, Mélanie Courtot, Dirk Derom, Jennifer M Fostel, Yongqun He, Phillip Lord, James Malone, Helen Parkinson, Bjoern Peters, and Philippe Rocca-Serra. 2010. "Modeling biomedical experimental processes with OBI." Journal of Biomedical Semantics 1 (1): 7.

[36] Ashino, Toshihiro. 2010. "Materials ontology: An infrastructure for exchanging materials information and knowledge." Data Science Journal 9:54-61.

[37] Watari, Fumio, Atsuro Yokoyama, Fuminori Saso, Motohiro Uo, and Takao Kawasaki. 1997. "Fabrication and properties of functionally graded dental implant." Composites Part B: Engineering 28 (1-2):5-11.

[38] Khor, KA, and YW Gu. 2000. "Thermal properties of plasma-sprayed functionally graded thermal barrier coatings.” Thin Solid Films 372 (1-2):104-113.

[39] Bandrowski, Anita, Ryan Brinkman, Mathias Brochhausen, Matthew H Brush, Bill Bug, Marcus C Chibucos, Kevin Clancy, Mélanie Courtot, Dirk Derom, and Michel Dumontier, et al. 2016. "The Ontology for Biomedical Investigations." PLoS one 11 (4):1-19.

[40] Tarlochan, Faris. 2012. "Functionally graded material: A new breed of engineered material.” Journal of Applied Mechanical Engineering.

[41] Zhou, MY, JT Xi, and JQ Yan. 2004. "Modeling and processing of functionally graded materials for rapid prototyping." Journal of Materials Processing Technology 146 (3):396-402.

[42] Zhu, Feng. 2004. "Visualized CAD modeling and layered manufacturing modeling for components made of a multiphase perfect material." HKU Theses Online (HKUTO). [43] Pasko, Alexander, Valery Adzhiev, Alexei Sourin, and Vladimir Savchenko. 1995. "Function representation in geometric modeling: concepts, implementation and applications." The Visual Computer 11 (8):429-446. 
[44] Chiu, WK, and ST Tan. 2000. "Multiple material objects: from CAD representation to data format for rapid prototyping." Computer-Aided Design 32 (12):707-717. [45] Heimann, Robert B. 2008. Plasma-spray coating: principles and applications: John Wiley \& Sons.

[46] Allahyarzadeh, MH, M Aliofkhazraei, AR Sabour Rouhaghdam, and V Torabinejad. 2016. "Gradient electrodeposition of Ni-Cu-W (alumina) nanocomposite coating."

Materials \& Design 107:74-81.

[47] Kumar, Sanjay, and Sisa Pityana. 2011. "Laser-based additive manufacturing of metals." Advanced Materials Research 227:92-95.

[48]Mumtaz, Kamran Aamir, and Neil Hopkinson. 2007. "Laser melting functionally graded composition of Waspaloy ${ }^{\circledR}$ and Zirconia powders." Journal of Materials Science 42 (18):7647-7656.

[49] Su, Wei-Nien. 2002. "Layered fabrication of tool steel and functionally graded materials with a Nd: YAG pulsed laser." PhD thesis.

[50] Soodi, Mehdi, SH Masood, and Milan Brandt. 2013. "Thermal expansion of functionally graded and wafer-layered structures produced by laser direct metal deposition." The International Journal of Advanced Manufacturing Technology 69 (912):2011-2018.

[51] Put, Stijn, Jef Vleugels, Guy Anné, and Omer Van der Biest. 2003. "Functionally graded ceramic and ceramic-metal composites shaped by electrophoretic deposition." Colloids and Surfaces A: Physicochemical and Engineering Aspects 222 (1-3):223-232. [52] Yongming, Luo, Pan Wei, Li Shuqin, Wang Ruigang, and Li Jianqiang. 2003. "A novel functionally graded material in the Ti-Si-C system." Materials Science and Engineering: A 345 (1-2):99-105.

[53] Rajan, TPD, RM Pillai, and BC Pai. 2010. "Characterization of centrifugal cast functionally graded aluminum-silicon carbide metal matrix composites." Materials Characterization 61 (10):923-928.

[54] Gupta, Ankit, and Mohammad Talha. 2015. "Recent development in modeling and analysis of functionally graded materials and structures." Progress in Aerospace Sciences 79:1-14.

[55] Naebe, Minoo, and Kamyar Shirvanimoghaddam. 2016. "Functionally graded materials: A review of fabrication and properties." Applied Materials Today 5:223-245. [56] Shanmugavelayutham, Gurusamy, and Akira Kobayashi. 2007. "Mechanical properties and oxidation behaviour of plasma sprayed functionally graded zirconiaalumina thermal barrier coatings." Materials Chemistry and Physics 103 (2-3):283-289. [57] Latiff, Muhammad Ihsan Abdul, Siti Nur Sakinah Jamaludin, Shahnor Basri, Ahmad Hussain, Dheya Shuja Alothmany, Faizal Mustapha, Dewan Muhammad Nuruzzaman, Noor Mazni Ismail, and Izwan Ismail. 2014. "Effect of Sintering Temperature on Functionally Graded Nickel/Alumina Plate.” Applied Mechanics and Materials 629:437443. 
[58] Kırmızı, Görkem, Halil Arık, and Henifi Çinici. 2019. "Experimental study on mechanical and ballistic behaviours of silicon carbide reinforced functionally graded aluminum foam composites." Composites Part B: Engineering 164:345-357.

[59] Mahmoud, Dalia, and Mohamed Elbestawi. 2017. "Lattice structures and functionally graded materials applications in additive manufacturing of orthopedic implants: a review.” Journal of Manufacturing and Materials Processing 1 (2):13. 